\title{
The population genetics of the self- incompatibility polymorphism in Papaver rhoeas. X. An association between incompatibility genotype and seed dormancy
}

\author{
M. D. LANE \& M. J. LAWRENCE* \\ Wolfson Laboratory for Plant Molecular Biology, School of Biological Sciences, University of Birmingham, \\ Birmingham B15 2TT, U.K.
}

\begin{abstract}
Further analysis of data presented in a previous paper (Lane \& Lawrence, 1995) has revealed an association between incompatibility genotype and seed dormancy such that the seed of crosses in which the wild-type allele, $S_{10}$, was segregating was a little less dormant, and those in which $S_{11}$ was segregating was slightly more dormant, than the average for the families in which these crosses occurred. It is argued that this association is caused by the linkage of the $S$-locus to one or more genes that determine seed dormancy in this species and that this linkage is one possible cause of the unequal $S$-allele frequencies observed in the natural population from which the wild-type parents of these progenies were obtained.
\end{abstract}

Keywords: Papaver rhoeas, seed dormancy, self-incompatibility.

\section{Introduction}

Lawrence \& Franklin-Tong (1994) observed disturbed segregation ratios for incompatibility genotype in two full-sib families of wild-type Papaver rhoeas, whose members had been raised from seed whose dormancy had been only partially broken. They suggested that the disturbed ratios in these families was the result of linkage of the self-incompatibility $(S-)$ gene to one or more genes that control seed dormancy and that this linkage might be one of the causes of the unequal $S$ allele frequencies in natural populations of the species (Campbell \& Lawrence, 1981; Lawrence \& O'Donnell, 1981). We showed in a previous paper that a genetic model containing six parameters specifying additive, dominance and epistatic effects accounted satisfactorily for variation in seed dormancy between the means of families of the basic generations $\left(\mathrm{P}_{1}, \mathrm{P}_{2}, \mathrm{~F}_{1}\right.$, $\mathrm{F}_{2}, \mathrm{~B}_{1}, \mathrm{~B}_{2}$ ) of a cross between wild-type $P$. rhoeas and its 'Shirley' cultivar, seed of the latter lacking the dormancy of the former (Lane \& Lawrence, 1995). There was also considerable variation within these families for this character. The wild-type parents of these families came from one of the families investigated by Lawrence \& Franklin-Tong (1994). It is possible, therefore, that the variation within the families examined by Lane \& Lawrence (1995) may *Correspondence. have resulted from variation among the self-incompatibility genotypes of their members. The selfincompatibility genotype of the parents of these families had been determined in the usual way (Lawrence, 1975; Lawrence et al., 1978, 1993) before they were crossed for seed, so that the composition of these families with respect to the incompatibility alleles segregating within them could be predicted. The data presented in the previous paper can be used, therefore, to investigate the possibility of an association between seed dormancy and self-incompatibility genotype; this is the purpose of the analysis given in the present paper.

\section{Materials and methods}

The 'Shirley' family used in this investigation was segregating for three $S$-alleles and the R102 wild-type Family 9 for four (Lawrence \& Franklin-Tong, 1994). Crosses were made using plants of all three of the possible incompatibility genotypes of the 'Shirley' family $\left(\mathrm{P}_{1}\right)$ and all six genotypes of the wild-type family $\left(\mathrm{P}_{2}\right)$. With three alleles in one parental family and four different alleles in the other, a total of 12 genotypes is expected in their $F_{1}$ progenies. It was discovered in the previous season, however, that one of the 'Shirley' alleles, referred to by Lawrence \& Franklin-Tong (1994) as $S_{3}$, was the same as the R102 allele, $S_{11}$, of 
Family 9. Because we wished to ensure that all progenies in the experiment, apart from those within the 'Shirley' cultivar, were of the four-class kind, only the 'Shirley' genotype which lacked the $S_{3}$ allele was used as a parent to produce the $F_{1}$ families. Hence, only eight, rather than 12 , genotypes occurred among the $F_{1}$ families. Lawrence \& Franklin-Tong (1994) referred to the alleles of the 'Shirley' family as $S_{1}, S_{2}$ and $S_{3}$. It is convenient, however, to refer to these 'Shirley' alleles as $S_{51}, S_{52}$ and $S_{53}$, respectively, to retain the original labelling of the R102 alleles of Family 9 as $S_{1}, S_{5}, S_{10}$ and $S_{11}$ (Lawrence \& O'Donnell, 1981).

A 'Shirley' plant whose genotype was $S_{51} S_{52}$ was crossed to each of two wild-type plants whose genotypes were $S_{1} S_{11}$ and $S_{5} S_{10}$ to produce two $F_{1}$ families and their reciprocals. Two sets of $17 \times 17$ diallel crosses were then made in the following season. The parents of the first set included eight plants from the first of these $F_{1}$ families, one of each of the three genotypes from the 'Shirley' family and one of each of the six genotypes from the wild-type family. The second set was the same as the first except that eight plants from the second $F_{1}$ family replaced those from the first $F_{1}$ family. All possible crosses giving rise to four-class families were made between the 17 plants of each set, including reciprocals, except in the case of crosses between 'Shirley' plants inter se where this was not possible, so that half-compatible crosses were made instead. Crosses that occurred in both sets were made only in the first. The crosses made between the plants of the first set are shown in Fig. 1 and those between the plants of the second set in Fig. 2.

\section{Results}

No family produced a set of crosses which contained all 20 incompatibility genotypes (15 distinct genotypes plus five more allowing for the different origins of the functionally identical alleles, $S_{11}$ and $S_{53}$ ) and no pair of families contained the same set of crosses, so that orthogonal comparisons between either $S$-alleles or $S$ genotypes cannot be extracted from the data. The possibility of an association between particular $S$ alleles and seed dormancy was, therefore, investigated in the following way. Consider a set of crosses belonging to a particular family, $B_{2\{(2 \times 1) \times 2\}}$ say, from each of which a germination percentage of the seed has been obtained (Lane \& Lawrence, 1995). After angular transformation of the germination data, a set of deviations, $d_{\mathrm{i}}$, was obtained by subtracting the family mean from the score of each of the 44 crosses (capsules) in the family. A table was then constructed for each family

\begin{tabular}{|c|c|c|c|c|c|c|c|c|c|c|c|c|c|c|c|c|c|c|}
\hline \multirow[t]{2}{*}{ Parents } & \multicolumn{4}{|c|}{$P_{1}$} & \multicolumn{4}{|c|}{$F_{1(1 \times 2)}$} & \multicolumn{4}{|c|}{$F_{1(2 \times 1)}$} & \multicolumn{6}{|c|}{$P_{2}$} \\
\hline & $S_{S_{1}} S_{1}$ & 51,52 & 51,53 & 52,53 & 1,51 & 1,52 & 11,51 & 11,52 & 1,51 & 1,52 & 11,51 & 11,52 & 1,5 & 1,10 & 1,11 & 5,10 & 5,11 & 10,11 \\
\hline \multirow{3}{*}{$P_{1}$} & 51,52 & . & 1 & $\checkmark$ & . & & . & . & . & . & . & . & 1 & $\checkmark$ & $\checkmark$ & 1 & $\checkmark$ & $\checkmark$ \\
\hline & 51,53 & $\checkmark$ & . & 1 & . & 1 & & . & . & $\checkmark$ & . & . & 1 & $\checkmark$ & . & $\checkmark$ & . & . \\
\hline & 52,53 & 1 & 1 & . & 1 & . & . & . & 1 & . & & . & 1 & $\checkmark$ & . & 1 & . & . \\
\hline \multirow{4}{*}{$F_{1(1 \times 2)}$} & 1,51 & . & . & $\checkmark$ & . & . & & $\checkmark$ & . & . & . & $\checkmark$ & . & . & . & $\checkmark$ & $\checkmark$ & $\checkmark$ \\
\hline & 1,52 & & $\checkmark$ & . & . & . & $\checkmark$ & . & . & . & 1 & . & . & . & . & $\checkmark$ & $\checkmark$ & 1 \\
\hline & 11,51 & . & . & . & . & 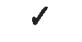 & & . & . & 5 & & . & $\checkmark$ & $\checkmark$ & . & $\checkmark$ & 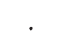 & . \\
\hline & 11,52 & . & . & . & 1 & . &. & . & 1 & . & . & . & 1 & $\checkmark$ & . & 1 & . & . \\
\hline \multirow{4}{*}{$F_{1(2 \times 1)}$} & 1,51 & . & . & $\checkmark$ & . & . & & $\checkmark$ & . & . & . & $\checkmark$ & . & . & . & 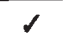 & $\checkmark$ & $\checkmark$ \\
\hline & 1,52 & . & $\checkmark$ & . & . & . & $\checkmark$ & . & . & . & $\checkmark$ & . & . & . & . & $d$ & $\checkmark$ & $\checkmark$ \\
\hline & 11,51 & . & . & . & . & 1 & . & . & . & 1 & . & . & $\checkmark$ & 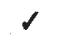 & . & $\checkmark$ & . & ' \\
\hline & 11,52 & . & . & $\dot{ }$ & 8 & &. & . & 1 & . &. &. & 1 & $\checkmark$ & . & 1 & . & . \\
\hline \multirow{6}{*}{$P_{2}$} & 1,5 & $\checkmark$ & $\checkmark$ & 1 & $\cdot$ & . & $\checkmark$ & $\checkmark$ & r & . & 1 & $\checkmark$ & . & . & & . & . & $\checkmark$ \\
\hline & 1,10 & $\checkmark$ & $\checkmark$ & 1 & . & . & $\checkmark$ & $\checkmark$ & . & . & $\checkmark$ & $\checkmark$ & . & . & . & . & $\checkmark$ & . \\
\hline & 1,11 & 1 & . & . & . & . & & . & $\cdot$ & . & . & . & . & . & 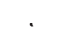 & 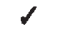 & . & . \\
\hline & 5,10 & $\checkmark$ & 1 & $\checkmark$ & $\checkmark$ & $\checkmark$ & 1 & $\checkmark$ & $\checkmark$ & $\checkmark$ & $\checkmark$ & $\checkmark$ & . & . & $\checkmark$ & . & & . \\
\hline & 5,11 & $\checkmark$ & . & . & $\checkmark$ & $\checkmark$ & . & . & $\checkmark$ & $\checkmark$ & . & . & . & $\checkmark$ & & $\cdot$ & $\cdot$ & . \\
\hline & 10,11 & 1 & . & . & 1 & 1 & 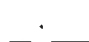 & . & 1 & 1 & & & $\checkmark$ & . & . & . & & . \\
\hline
\end{tabular}

Fig. 1 First set of diallel crosses for Papaver rhoeas, showing the incompatibility genotypes of the parents crossed to produce the progenies, indicated by a tick, shown in the body of the figure. $P_{1}$ is the 'Shirley' and $P_{2}$ the wild-type parent. Although functionally identical, it is convenient to distinguish between the 'Shirley' allele, $S_{53}$, and the wild-type allele, $S_{11}$, because of their different origins. 


\begin{tabular}{|c|c|c|c|c|c|c|c|c|c|c|c|c|c|c|c|c|c|c|}
\hline \multirow[t]{2}{*}{\begin{tabular}{|l|} 
Parents \\
\end{tabular}} & \multicolumn{4}{|c|}{$P_{1}$} & \multicolumn{4}{|c|}{$F_{1(1 \times 2)}$} & \multicolumn{4}{|c|}{$F_{1(2 \times 1)}$} & \multicolumn{6}{|c|}{$P_{2}$} \\
\hline & $S_{S_{1}} S_{1}$ & 51,52 & 51,53 & 52,53 & 5,51 & 5,52 & 10,51 & 10,52 & 5,51 & 5,52 & 10,51 & 10,52 & 1.5 & 1,10 & 1,11 & 5,10 & 5,11 & 10,11 \\
\hline \multirow{3}{*}{$P_{1}$} & 51,52 & . & . & . & ' & . & & & . & & . & . & 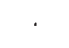 & . & . & . & . & \\
\hline & 51,53 & . & . & . & & $\checkmark$ & & $\checkmark$ & . & $\checkmark$ & . & $\checkmark$ & . & . & . & . & . & \\
\hline & 52,53 & . & . & . & 1 & . & 1 & . & 1 & . & 1 & . & & . & . & $\therefore$ & 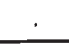 & . \\
\hline \multirow{4}{*}{$F_{1(1 \times 2)}$} & 5,51 & . & . & $\checkmark$ & . & . & . & $\checkmark$ & & . & . & $\checkmark$ & . & $\checkmark$ & $\checkmark$ & . & . & $\checkmark$ \\
\hline & 5,52 & . & $\checkmark$ & . & . & . & $\checkmark$ & . & . & . & $\checkmark$ & . & . & $\checkmark$ & $\checkmark$ & . & . & $\checkmark$ \\
\hline & 10,51 & . & . & $\checkmark$ & . & 8 & . & & & $\checkmark$ & . & . & 8 & . & $\checkmark$ & . & $\checkmark$ & . \\
\hline & 10,52 &. & 1 & . & $\checkmark$ & . & & . & 1 & . & . & . & 1 & . & 1 & . & 1 &. \\
\hline \multirow{4}{*}{$F_{1(2 \times 1)}$} & 5,51 & 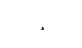 & . & $\checkmark$ & . & . & . & $\checkmark$ & & . & . & $\checkmark$ & . & $\checkmark$ & $\checkmark$ & . & . & $\checkmark$ \\
\hline & 5,52 & . & $\checkmark$ & & & . & $\checkmark$ & & . & . & $\checkmark$ & . & . & $\checkmark$ & $\checkmark$ & . & & $\checkmark$ \\
\hline & 10,51 & . & & $\checkmark$ & . & $\checkmark$ & . & . & . & $\checkmark$ & & . & $\checkmark$ & . & $\checkmark$ & . & $\checkmark$ & . \\
\hline & 10,52 & . & 1 & . & 1 & . & . & . & 1 &. & & . & 1 &. & $\checkmark$ &. & $\checkmark$ & . \\
\hline \multirow{6}{*}{$P_{2}$} & 1,5 & . & . & . & . & . & $\checkmark$ & $\checkmark$ & . & . & $\checkmark$ & $\checkmark$ & . & . & . & & . & \\
\hline & 1,10 & . & . & . & $\checkmark$ & $\checkmark$ & . & & $\checkmark$ & $\checkmark$ & . & & . & . & . & . & . & . \\
\hline & 1,11 & . & . & . & $\checkmark$ & 8 & $\checkmark$ & 1 & $\checkmark$ & $\checkmark$ & $\checkmark$ & $\checkmark$ & . & . & . & . & . & . \\
\hline & 5,10 & . & . & . & . & . & . & & . & . & . & . & . & . & & . & . & . \\
\hline & 5,11 & . & . & . & . & & $\checkmark$ & $\checkmark$ & . & . & $\checkmark$ & $\checkmark$ & . & . & . & . & . & . \\
\hline & 10,11 & . & . & . & 1 & 1 & . & . & 1 & $\checkmark$ & . & & . & . & . & & . & \\
\hline
\end{tabular}

Fig. 2 Second set of diallel crosses for Papaver rhoeas. Crosses common to both sets were made only in the first.

in which the deviation of each cross was assigned to each of the $S$-alleles predicted to be segregating in that cross from a knowledge of the incompatibility genotypes of its parents. A mean deviation, $d_{\mathrm{i}}$, for each of the $S$-alleles segregating in the family was, thereafter, obtained by summing deviations over crosses and dividing this sum by the number of crosses in which the $S$-allele in question occurred. Standard errors of these mean deviations were derived from the variance of the individual deviations about their mean and used to carry out either a normal deviate or $t$-test, according to their degrees of freedom, to test the significance of the departure of these deviations from zero.

There are four points that need to be made about this analysis. First, the data from the small number of crosses of the 'Shirley' family were omitted because, being of the two-class kind, their inclusion would have complicated the analysis considerably for a rather small gain in information. Secondly, families in which all crosses contain the same two or three $S$-alleles (all crosses in the $\mathrm{F}_{2}$ families segregated for $S_{51}$ and $S_{52}$, and all in $\mathrm{B}_{1}$ contained all three 'Shirley' alleles) cannot provide any information about an association between seed dormancy and these alleles, because their mean deviations must equal zero. Thirdly, it is not possible to compare directly the effects of the 'Shirley' $S$-alleles on seed dormancy with those of the wild-type alleles because all crosses within a family necessarily have the same composition with respect to these two sources of
$S$-alleles. Comparisons can, however, be made between the 'Shirley' alleles inter se and the wild-type alleles inter se. Lastly, although the mean deviations of $S$ alleles from different families are independent, those from the same family are not.

The results obtained by averaging the mean deviations of the $S$-alleles over the families of the experiment (Table 1) show that whereas, as expected, none of the deviations of the 'Shirley' alleles differs significantly from zero, those of two of the wild-type alleles do, the positive deviation of $S_{10}$ suggesting that the seed of crosses in which this allele is present is a little less dormant than average and the negative deviation of $S_{11}$ indicating the reverse.

The results obtained from each family are shown in Table 2. Inspection of the entries in this table reveals that nine pairs of deviations are completely correlated, which arises from the fact that, for example, all $F_{2}$ families contain either $S_{1}$ and $S_{11}$ or $S_{5}$ and $S_{10}$, so that it is necessary to exercise some caution in interpreting these results. That said, four of the deviations shown in the table differ significantly from zero, two concerning $S_{1}$ and two $S_{11}$. The pair of significant deviations associated with $S_{1}$, however, cannot be taken seriously for three reasons. First, while one of these deviations is positive, the other is negative. Secondly, the latter is completely correlated with the deviation associated with $S_{11}$ in this $F_{2}$ family; it can be argued, therefore, that its significance results from this statistical 
relationship alone. Thirdly, of the 14 deviations associated with $S_{1}$ in Table 2, six are positive and eight are negative, indicating that this allele is not associated with any consistent effect on seed dormancy. The significance of the single positive deviation concerning this allele is, therefore, almost certainly the result of chance alone.

The pair of significant deviations concerning $S_{11}$, however, are not only of the same sign, but are two of no less than nine negative deviations out of a total of 10 , which is a significant departure on a sign test from the expected equality of positive and negative devia-

Table 1 Mean deviations of seed germination (after angular transformation), averaged over families, for the four wildtype $S$-alleles $\left(S_{1}, S_{5}, S_{10}, S_{11}\right)$ of Papaver rhoeas and the three 'Shirley' alleles $\left(S_{51}, S_{52}, S_{53}\right)$ that were segregating in the crosses of the experiment

\begin{tabular}{rcrc}
\hline$S_{\mathrm{i}}$ & No. of crosses & Mean deviation $\left(d_{\mathrm{i}}\right)$ & $P$ \\
\hline 1 & 186 & $0.59 \pm 1.28$ & $0.55-0.54$ \\
5 & 174 & $0.11 \pm 1.17$ & $0.93-0.92$ \\
10 & 168 & $3.09 \pm 1.31$ & $0.02-0.01^{*}$ \\
11 & 146 & $-4.44 \pm 1.32$ & $<0.001^{* * *}$ \\
51 & 94 & $0.31 \pm 1.85$ & $0.64-0.63$ \\
52 & 106 & $-0.06 \pm 1.63$ & $0.87-0.86$ \\
53 & 24 & $-0.98 \pm 2.07$ & $0.98-0.97$ \\
\hline
\end{tabular}

The number of crosses on which each deviation is based is shown in column two and the probability, from the normal deviate test, that the estimate differs from zero is shown in the fourth column. See the text for other details. tions on the null hypothesis of no association between $S$-genotype and seed dormancy. These results suggest, therefore, that this allele is associated with an effect on seed dormancy, a conclusion which is, of course, consistent with that drawn from the results obtained by pooling the data over families (Table 1).

None of the deviations concerning $S_{10}$, however, which from the combined analysis also appeared to be associated with an effect on seed dormancy, is significant. On the other hand, 11 of a total of 14 of these deviations are positive, which is a significant departure from equality on the sign test. Hence, while the results for this allele are, perhaps, somewhat less persuasive than those of $S_{11}$, they are, nevertheless, consistent with those obtained from the analysis of the data combined over families. The chief point we wish to make here is that the significant deviations associated with $S_{10}$ and $S_{11}$ in the combined analysis are not simply the result of one or two large family deviations, but reflect a degree of consistency in the sign of these deviations over the families of the experiment, those associated with $S_{10}$ being, in general, positive and those associated with $S_{11}$ being, in general, negative. In contrast, there is no evidence of any consistent association between either the wild-type alleles, $S_{1}$ and $S_{5}$, or the 'Shirley' alleles, $S_{51}, S_{52}$ and $S_{53}$, and seed dormancy.

\section{Discussion}

The chief causes of the less than wholly satisfactory analysis that has had to be employed with these data are that the 'Shirley' family was segregating for only

Table 2 Mean deviations of seed germination (after angular transformation) for $S$-alleles in each family of Papaver rhoeas

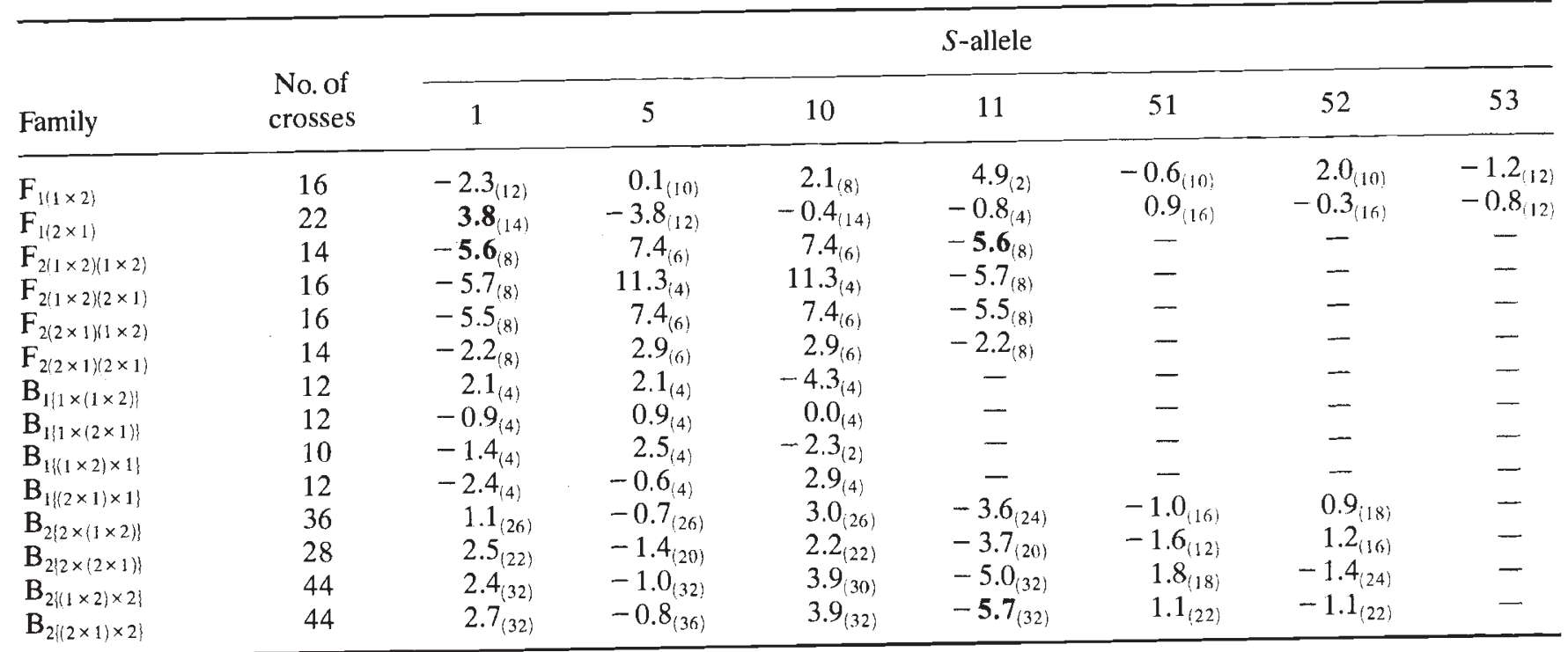

Subscripts attached to the deviations indicate the number of occurrences of each $S$-allele in each family. The deviations shown in bold differ significantly $(P=0.05-0.01)$ from zero.

(c) The Genetical Society of Great Britain, Heredity, 75, 92-97. 
three, rather than four alleles and that, in particular, one of these alleles turned out to be the same as one of those segregating in the wild-type family. At the time that this experiment was initiated, the 'Shirley' family used was the only one about which sufficient was known for this purpose, although others have since been produced, including some in which four alleles are segregating. The discovery that the 'Shirley' allele, referred to here as $S_{53}$, was functionally identical to the wild-type allele, $S_{11}$, however, was quite unexpected, not only because this allele occurs at a relatively high frequency in none of the British natural populations we have examined (Lawrence et al., 1993), but also because, the 'Shirley' cultivar marketed by Suttons, from which this family was derived (Lawrence \& Franklin-Tong, 1994; Lane \& Lawrence, 1995), appears to contain, as might be expected, a smaller number of alleles $(\hat{N}=18)$ than natural populations (Lane, 1990).

In principle, it would of course be possible to design an experiment which avoided these problems, by ensuring that the 'Shirley' family was of the four-class type and possessed no alleles that also occurred in the wild-type family used in the experiment. If these conditions were met, it should be possible to extract orthogonal comparisons between alleles in respect of their association with seed dormancy, because each of these alleles would be equally represented among the parents and hence equally represented among their progeny. The size of such an experiment, however, would be very large. Thus, with four alleles in each parent, there would be 16 different $F_{1}$ genotypes which, if crosses between parents were made in reciprocal, would give a total of 44 genotypes in all ( $32 \mathrm{~F}_{1}$ genotypes and six of each of $P_{1}$ and $\left.P_{2}\right)$ and, hence, entail a $44 \times 44$ set of diallel crosses. Even if, as in the present experiment, crosses were confined to those giving rise to four-class families, this would still require the production and testing of the seed of no less than 948 crosses. It is very doubtful whether an experiment of this size could be carried out on plants raised in the open, chiefly because by the time 44 parents of the desired incompatibility genotype had been identified, the plants concerned would be too old to yield good seed. Hence, in practice, any experiment of realistic size that was designed with the same objective would be unlikely to be much more complete than the present one.

Despite these reservations about the efficiency of the analysis it has, nevertheless, been possible to detect an association between two of the wild-type alleles and seed dormancy, the seed of crosses in which $S_{10}$ is present being a little less dormant and that of those in which $S_{11}$ is present being more dormant than average; that is, these alleles appear to be subject to an extra effect of selection over and above that arising from incompatibility. In principle, this additional selection could result from pleiotropy or linkage to one or more genes that are the chief target of this selection. Apart from the difficulty of seeing how an $S$-allele could be involved with the determination of seed dormancy as well as incompatibility, we have pointed out elsewhere that the fact that different $S$-alleles occur at relatively high frequencies in different natural populations is inconsistent with the notion that the unequal $S$-allele frequencies in these populations are caused by a pleiotropic property of these alleles (Lawrence et al., 1993). The best and most direct evidence on this possibility, however, comes from the present experiment. Thus, whereas $S_{11}$ appears to be associated with seed dormancy, the 'Shirley' allele, $S_{53}$, which is functionally identical to this wild-type allele, is not. Hence, the extra effect of selection on this allele must result from its linkage to one or more genes that control seed dormancy and the same is presumably true of that acting on the other wild-type allele, $S_{10}$.

This deduced linkage is, presumably, quite fortuitous for there is no obvious reason why genes concerned with two quite different aspects of the phenotype should be linked. It must, nevertheless, be quite tight, not only because it has been possible to detect it despite the inefficiency of the present analysis, but also because the strong directional dominance of the genes controlling seed dormancy (Lane \& Lawrence, 1995) must have reduced this efficiency still further. This investigation, therefore, appears to provide a possible explanation of the unequal $S$-allele frequencies in the R102 natural population, particularly because these results suggest that the frequency of $S_{10}$ (associated with lower than average dormancy) should be higher than that of $S_{11}$ (associated with higher than average dormancy) in this population which is, indeed, the case (Lawrence \& O'Donnell, 1981; Lawrence et al., 1993). The results of the present experiment are not consistent, however, with those of previous experiments. Thus, while there was clear evidence of disturbed segregation ratios with respect to incompatibility genotype in Family 9, from which the wild-type parents of the present investigation were obtained, this arose solely from an excess of plants containing $S_{1}$, one of the most frequently occurring alleles in the R102 population, relative to those carrying the alternative allele, $S_{5}$ (Lawrence \& Franklin-Tong, 1994). Furthermore, as this disturbance was confined to reciprocal A, the extra effect of selection acting on $S_{\mathrm{l}}$, over and above that resulting from incompatibility, appeared to be of the gametic type. But any extra effect of selection acting on the alleles of the $S$-locus because of their linkage to genes controlling seed dormancy would be of the zygotic, rather than the gametic type. While it is possible to accept that the 
extra effect of selection acting on $S_{1}$ may be of a different kind to that acting on $S_{10}$ and $S_{11}$, if the linkage between these alleles and the genes determining seed dormancy is as tight as the results of the present experiment suggest, we should have been able to detect this linkage in the form of disturbed segregation ratios in Family 9, particularly because the germination of the seed from which the plants of this family were raised was incomplete (Lawrence \& Franklin-Tong, 1994). As the ratio of plants containing $S_{10}$ to those containing $S_{11}$, obtained by pooling the data over Families 9 and 9.0, was 89:88 (Lawrence \& Franklin-Tong, 1994, tables 3 and 5), there is no evidence to support this retrospective prediction.

The present experiment was undertaken in an attempt to overcome the difficulties experienced in previous experiments when trying to control seed germination by pretreatment of seed, with the idea that this might be better accomplished by genetic, rather than environmental means. To the extent that it has provided information both on the genetic architecture of seed dormancy in the cross between 'Shirley' and wild-type $P$. rhoeas (Lane \& Lawrence, 1995) and on a previously suspected association between seed dormancy and incompatibility genotype (Lawrence \& Franklin-Tong, 1994), it can be regarded as successful. Although it can be argued that the conditions of the present experiment differed from those of previous experiments, this is a less than satisfactory explanation of the lack of repeatability between experiments. We are left, therefore, with the conclusion that while the evidence from these experiments suggests that the $S$ locus is subject to an extra effect of selection, over and above that concerned with incompatibility, and that this is a likely cause of the unequal $S$-allele frequencies observed in the natural populations we have examined, this selection is of several different kinds, of which that caused by linkage between the $S$-locus and genes controlling seed dormancy is only one.

\section{Acknowledgements}

The work described in this paper was supported by the award of an NERC studentship to M.D.L. and an NERC research grant to M.J.L. which are gratefully acknowledged.

\section{References}

CAMPBell, J. M. AND LAWRENCE, M. J. 1981. The population genetics of the self-incompatibility polymorphism in Papaver rhoeas. II. The number and frequency of $S$-alleles in a natural population (R106). Heredity, 46, 81-90.

LANE, M. D. 1990. The Population Genetics of the Selfincompatibility Polymorphism in Papaver rhoeas. Ph.D. Thesis, University of Birmingham.

LANE, M. D. AND LAWRENCE, M. J. 1995. The genetics of seed dormancy in Papaver rhoeas. Heredity, 75, 84-91.

LAWRENCE, M. J. 1975. The genetics of self-incompatibility in Papaver rhoeas. Proc. R. Soc. B, 188, 275-285.

LAWRENCE, M. J., AFZAL, M. AND KENRICK, J. 1978. The genetical control of self-incompatibility in Papaver rhoeas. Heredity, 40, 239-253.

LAWRENCE, M. J. AND FRANKLIN-TONG, V. E. 1994. The population genetics of the self-incompatibility polymorphism in Papaver rhoeas. IX. Evidence of an extra effect of selection acting on the $S$-locus. Heredity, 72, 353-364.

LAWRENCE, M. J., LANE, M. D., O'DONNELL, S. AND FRANKLIN-TONG, V. E. 1993. The population genetics of the self-incompatibility polymorphism in Papaver rhoeas. V. Cross-classification of the $\mathrm{S}$-alleles of samples from three natural populations. Heredity, 71, 581-590.

LAWRENCE, M. J. AND O'DONNELL, s. 1981. The population genetics of the self-incompatibility polymorphism in Papaver rhoeas. III. The number and frequency of $S$ alleles in two further natural populations (R102 and R104). Heredity, 47, 53-61. 